Gerhard Stickel

Mannheim

\title{
Einleitung zum Podiumsgespräch über „Zugänge zum Text“6
}

Die bisherigen Beiträge zu diesem Kolleg haben wieder einmal deutlich gemacht, wie weit und aspektreich der Gegenstandsbereich all dessen ist, was sich unter der Bezeichnung Text subsumieren lässt: von der knappen Aufforderung Komm bis beispielsweise zu Thomas Manns Roman Josef und seine Brüder auf fast 2000 Druckseiten oder gar Marcel Prousts $A$ la recherche du temps perdu, von der Aufschrift auf dem Schild eines Zahnarztes bis zum Schlussplädoyer eines Staatsanwalts, von den graphematischen Eigenschaften eines Briefs bis zu den Intonationskonturen eines Streitgesprächs. Wir fünf hier auf dem Podium werden erst gar nicht versuchen, in bloß anderthalb Stunden die Fülle und Vielfalt des Phänomenbereichs , $\mathrm{Text}^{\mathrm{t}}$, so wie er in den vielen Vorträgen präsentiert wurde, unter einen Hut zu bringen. Das heißt: erwarten Sie bitte kein Resümee oder eine Konklusion aus der gesamten bisherigen Tagung. In der Hoffnung, zumindest das eine oder andere bisher diskutierte Motiv wieder aufnehmen zu können, möchten wir uns darauf beschränken, das uns gestellte Thema „Zugänge zum Text " in einer seiner möglichen Lesarten arbeitsteilig zu behandeln. Das heißt, wir wollen einander und damit auch den anderen Tagungsteilnehmern kurz über den Umgang mit geschriebenen Texten berichten, wie er für die Literaturwissenschaft, die Rechtswissenschaft und die Linguistik charakteristisch ist. Dabei werden wir besonders auf die Rezipienten von geschriebenen Texten eingehen. Zum Ablauf vielleicht so viel: Jeder von uns wird zunächst kurz aus der Sicht seines Fachs auf das Thema eingehen. In einer zweiten Runde kann dann Ergänzendes vorgetragen werden. Danach sollte möglichst bald die Gesprächsrunde geöffnet werden; das heißt, dann kann sich auch das verehrte Publikum mit Fragen, Meinungsäußerungen oder Zusatzinformationen beteiligen. Aber zunächst $\mathrm{zu}$ uns hier auf dem Podium

Lassen Sie mich bitte die ,Podianten“ kurz vorstellen:

- Frau Professor Orosz von der ELTE in Budapest und Herr Kollege Csúri von der Szegediner Universität vertreten die Literaturwissenschaft.

- Die beiden Universitätsdozenten Frau Karsai und Herr Szomora vom Institut für Strafrecht an der hiesigen Universität sind uns als Juristen willkommen.

- Ich bin Linguist und komme vom Institut für Deutsche Sprache in Mannheim. 
Nun zu unserem Thema „Zugänge zum Text“. Als wissenschaftliches Fach tut sich die Linguistik mit Texten meist recht schwer; denn sie sieht sich der ganzen Breite und Vielfalt von Texten gegenüber, die ich zu Beginn kurz angedeutet habe. Es gibt für die Linguistik keine ausgezeichneten Textaspekte oder Textsorten außer denen, die der einzelne Linguist für seine Arbeit ausgewählt hat. Im Unterschied zu den Kolleginnen und Kollegen in der Literaturwissenschaft haben Textlinguisten eine gewisse Präferenz für prosaische Gebrauchstexte, Zeitungsartikel und Alltagsgespräche. Dies wurde ja auch in mehreren der bisherigen Beiträge deutlich. Linguisten, die sich mit Texten als Menge wichtiger Erscheinungsformen von Sprache befassen, sind von den Texten, die sie untersuchen, zumeist nicht als Adressaten gemeint. Prinzipiell verhalten sich Linguisten Texten gegenüber extrakommunikativ. Sie können sich allenfalls probeweise in die Rezipientenrolle versetzen, um Texteigenschaften und Situationsbedingungen im Hinblick auf Hörer oder Leser zu analysieren. Ein gemeinsames Interessengebiet sehe ich für die Textlinguistik wie für die Literaturwissenschaft und die Rechtswissenschaft: es ist die Intertextualität, d.h. die Beziehung die viele Texte auf andere Texte haben durch direkte und indirekte Zitate, Verweise, thematische Anspielungen und Wiederaufnahmen. Hierüber könnte es zu einem fruchtbaren Austausch kommen.

Leicht hat es aber auch die Literaturwissenschaft nicht mit den Texten, zumal sie auch Texte zu berücksichtigen hat, die keine Entsprechungen in alltäglicher Kommunikation haben. Ich denke etwa an lyrische Gedichte. Immerhin kann sich die Literaturwissenschaft auf fiktionale Texte beschränken, also solche, die nicht für den alltäglichen Gebrauch intendiert sind. Zu den heiklen Fragen, die zu erörtern sind, gehört die nach den Unterscheidungsmerkmalen von fiktionalen und nichtfiktionalen Texten. Gibt es charakteristische textinterne strukturelle Eigenschaften, vielleicht eine spezifische Kohärenz? Wie steht es mit den Zwecken, die mit literarischen Texten intendiert sind? (Diese Frage ist vielleicht verboten, weil sie zu sehr an die altmodische Suche nach dem erinnert, was der Dichter wohl gemeint hat.) Vielleicht ist aber die moderne Literaturwissenschaft über die vage allgemeine Bestimmung dichterischer Zwecke hinausgelangt, die seit Horaz tradiert wird: aut prodesse aut delectare: nützen oder erfreuen oder beides. Doch wie steht es mit der Adressatenorientiertheit eines Romans oder eines Gedichts? Wie werden sie von wem rezipiert? Immerhin gibt es eine entwickelte Rezeptionsästhetik. Auch der einzelne Literaturwissenschaftler ist ja wie der so genannte normale Leser ebenfalls Rezipient literarischer Texte. Welche konzeptionellen und methodischen Zugänge zum jeweiligen Text hat sie, hat er im Unterschied zu laienhaften Literarturfreunden? Ich habe die Zuversicht, dass Frau Kollegin Orosz und Herr Kollege Csúri sich mit diesen und verwandten Fragen auseinandersetzen werden. 
Mit ihren Ausführungen werden vermutlich die Beiträge der beiden anderen Gesprächsteilnehmer kontrastieren, der beiden Dozenten für Rechtswissenschaft Frau Karsai und Herr Szomora. Damit möchte ich nicht auf die möglicherweise unterschiedlichen Vortragstile anspielen. Nein, es sind die Texte, mit denen sich die Vertreter der beiden Fächer befassen. Aus der Sicht der Textlinguistik sind Rechtstexte viel deutlicher konturiert als die vielen anderen Texte, denen wir im Alltag oder auch als Leser fiktionaler Literatur begegnen. Zugegeben, wenn man an die verschiedenen Texte denkt, mit denen Recht gesetzt, verordnet, vereinbart, gefordert, erörtert, erläutert und entschieden wird, erkennt man eine große Vielfalt von Textsorten. (Die Abfolge von Partizipien gesetzt, verordnet, vereinbart, gefordert, erörtert, erläutert und entschieden habe ich mir nicht für dieses Podium ausgedacht. Ich habe sie nach früheren Diskussionen mit Juristen aufgeschrieben.) Trotz dieser Vielfalt haben all die Texte im Rechtswesen eine gemeinsame Zweckbestimmung: Sie sollen Recht schaffen oder wieder herstellen. Als Texte in Funktion haben sie eine deutliche Handlungsqualität. Wie sich aus der vorbereitenden Korrespondenz ergibt, können wir von den beiden Juristen einen lehrreichen Überblick über Textsorten und Textverwendungen im Rechtswesen, speziell im Strafrecht erwarten und auch Hinweise auf die Intertextualität von Rechtstexten. Als naiver Linguist, der nur gelegentlich mit dem Sprachgebrauch von Juristen zu tun gehabt hat, würde ich Rechtstexte sicherlich zum prototypischen Kernbereich des gesamten Textfeldes rechnen, weil sie durchweg kohärent, klar abgegrenzt und funktional bestimmt sind.

Die folgenden schriftlichen Beiträge der beteiligten Literaturwissenschaftler und Juristen gehen über unser Szegediner Podiumsgespräch weit hinaus. Sie sind Ergebnis einer fruchtbaren Weiterarbeit der Beteiligten, die unser Gespräch zum Anlass genommen haben, ihre fachspezifischen „Zugänge zum Text" ausführlicher zu erläutern und zu begründen, als das bei unserem kurzen Zusammensein auf dem Podium möglich war. 